\title{
Quelle est la qualité de la recherche médicale?
}

\author{
Stefanie Hostettlera, Esther Kraft ${ }^{\mathrm{b}}$, Christoph Bosshard ${ }^{\mathrm{c}}$ \\ ${ }^{a}$ Dr sc. EPFZ, division DDQ; ${ }^{b}$ lic. rer. oec., cheffe division DDQ; ${ }^{\circ}$ Dr med., vice-président de la FMH, responsable du département DDQ/ASQM
}

\begin{abstract}
La division Données, démographie et qualité (DDQ) publie dans le Bulletin des médecins suisses les documents de base qu'elle établit sur différents thèmes relevant du domaine de la qualité. La FMH s'appuie sur ces réflexions pour déterminer son point de vue et le rendre officiellement public dans le document «Position de la FMH». Nous vous présentons ici le document de base ainsi que la prise de position de la FMH sur le thème «Recherche médicale: la qualité avant la quantité».
\end{abstract} sous www.bullmed.ch $\rightarrow$ Numéro actuel ou $\rightarrow$ Archives $\rightarrow 2015 \rightarrow 49$.
Les références se trouvent

\section{La recherche en pleine croissance}

Le volume mondial de publications a pratiquement triplé depuis le début des années 80 (1981-1985: 3,96 millions de publications; 2007-2011: 11,55 millions de publications).

Les Etats-Unis (28,2\%), la Chine (7,2\%) et l'Angleterre $(5,8 \%)$ produisent le plus grand nombre d'articles scientifiques dans le monde.

Avec 3,6 publications par an et 1000 habitants, la Suisse est championne du monde en nombre de publications par habitant. Par ailleurs, la contribution actuelle de la Suisse dans les domaines de la "Médecine clinique» et des "Life Sciences» est supérieure à la moyenne mondiale [1]*. En 2012, la Suisse a consacré, à elle seule, plus de 18 milliards de francs à la recherche et au développe-

\section{Résumé}

Dans la pratique médicale quotidienne, l'évidence scientifique est déterminante pour les décisions thérapeutiques, la formation prégraduée, postgraduée et continue et d'autres domaines médicaux. Ces derniers temps, la qualité de la recherche biomédicale a fait l'objet de critiques répétées et le nombre des rétractations d'études augmente. La fraude scientifique, les lacunes méthodologiques et statistiques d'études, les distorsions et la non-publication de résultats indésirables ainsi qu'une présentation incomplète et imprécise entravent la qualité de la recherche. Non seulement une recherche erronée représente un formidable gaspillage de ressources humaines et financières, mais conduit également à de fausses conclusions, et donc à une mise en danger de la sécurité des patients. Le présent rapport se penche sur les points faibles et les causes du manque de qualité de la recherche et met en évidence les possibilités d'agir. ment, dont deux tiers étaient financés par l'économie privée [2].

D'un côté, la promotion et le développement de la recherche soulignent l'importance de son rôle et constituent une base essentielle pour les innovations. De l'autre côté, il est indispensable de mener un débat critique sur les questions d'utilité et de qualité de la recherche, notamment en vue de la meilleure utilisation possible des ressources.

Depuis 2008, la communauté scientifique pointe du doigt les points faibles de la recherche. Plus récemment, l'opinion publique a commencé à émettre des doutes quant à la fiabilité et à l'indépendance de la recherche. Les médias parlent d'une crise de crédibilité [3] et d'un manque de confiance dans la science [4]. Le nombre des rétractations d'études est en augmentation [5]. L'exemple de l'étude menée par le Dr Andrew Wakefield qui révèle un lien entre le vaccin combiné rougeole-oreillons-rubéole (ROR) et l'autisme montre combien les conséquences d'études non correctement menées peuvent être graves: suite à la publication de l'étude en 1998 dans le Lancet, on a enregistré une recrudescence des cas d'oreillons parce que les parents inquiets ne faisaient plus vacciner leurs enfants. Lancet a retiré l'étude en 2010 pour méthodologie douteuse et fraude scientifique. Il n'existe à ce jour aucune preuve scientifique attestant d'un lien entre vaccin ROR et autisme. Hormis la violation des principes de l'intégrité scientifique (par ex. inventer ou falsifier des données, copier une propriété intellectuelle sans sources de référence, etc.), il existe d'autres facteurs influençant la qualité de la recherche biomédicale. Des enquêtes montrent par 
exemple que $85 \%$ des études contiennent des questions non pertinentes pour le patient [6,7], que $60 \%$ des résultats d'études ne sont pas reproductibles [8, 9], que presque $50 \%$ des études ne sont pas publiées $[10,11]$ et/ou que les publications contiennent souvent des informations lacunaires [12-15].

Le présent article se penche sur la qualité de la recherche biomédicale et met en évidence les sources d'erreurs possibles et leur contexte. Il a pour objectif de sensibiliser les médecins, les chercheurs et toute autre personne intéressée à la question de la qualité de la recherche, de faire connaître des outils concrets pour évaluer la qualité des études scientifiques et d'indiquer les possibilités d'agir.

\section{Facteurs d'influence négatifs sur la qualité de la recherche}

Dans le domaine de la recherche médicale, les études scientifiques servent par exemple à évaluer l'efficacité et la sécurité de mesures médicales ou à analyser d'autres questions (par ex. diagnostiques) sans réponse [16]. Divers facteurs tels que des fluctuations aléatoires, des mesures erronées ou des paramètres non pris en considération peuvent influencer les résultats.

La figure 1 décrit les principaux facteurs d'influence pouvant être évités qui ont un impact négatif sur la qualité de la recherche. Les différents facteurs peuvent en partie se chevaucher.

\section{Fraude scientifique}

On entend par fraude scientifique une violation des principes fondamentaux de la pratique scientifique. De gravité diverse, elle s'étend du non-respect des dispositions légales claires à la non-déclaration de conflits d'intérêts économiques ou individuels. Des exemples de fraude scientifique sont présentés à la figure 1 et détaillés dans la brochure Intégrité dans le domaine scientifique des Académies Suisses des Sciences [17].

La fraude n'est pas toujours délibérée, elle peut par exemple se produire lorsque les chercheurs travaillent dans un environnement axé sur la maximisation du profit. Palazzo et al. 2012 [18] entend par Ethical Blindness un comportement non délibéré et non éthique menant, dans l'activité de recherche, à une prise de décision, non pas objective, mais intuitive et automatique. Dans de telles circonstances, les scientifiques ne sont pas en mesure d'éviter un comportement non éthique. Souvent, ils prennent conscience de la fraude bien après et ils culpabilisent, même si ce sentiment diminue avec le temps.

\section{Distorsions (biais)}

On entend par biais une distorsion des résultats aux causes diverses. Les distorsions énumérées ci-après sont tirées de Jüni et al., 2001, Assessing the quality of randomised controlled trials [19], et font partie des formes de biais les plus connues dans le domaine de la recherche.

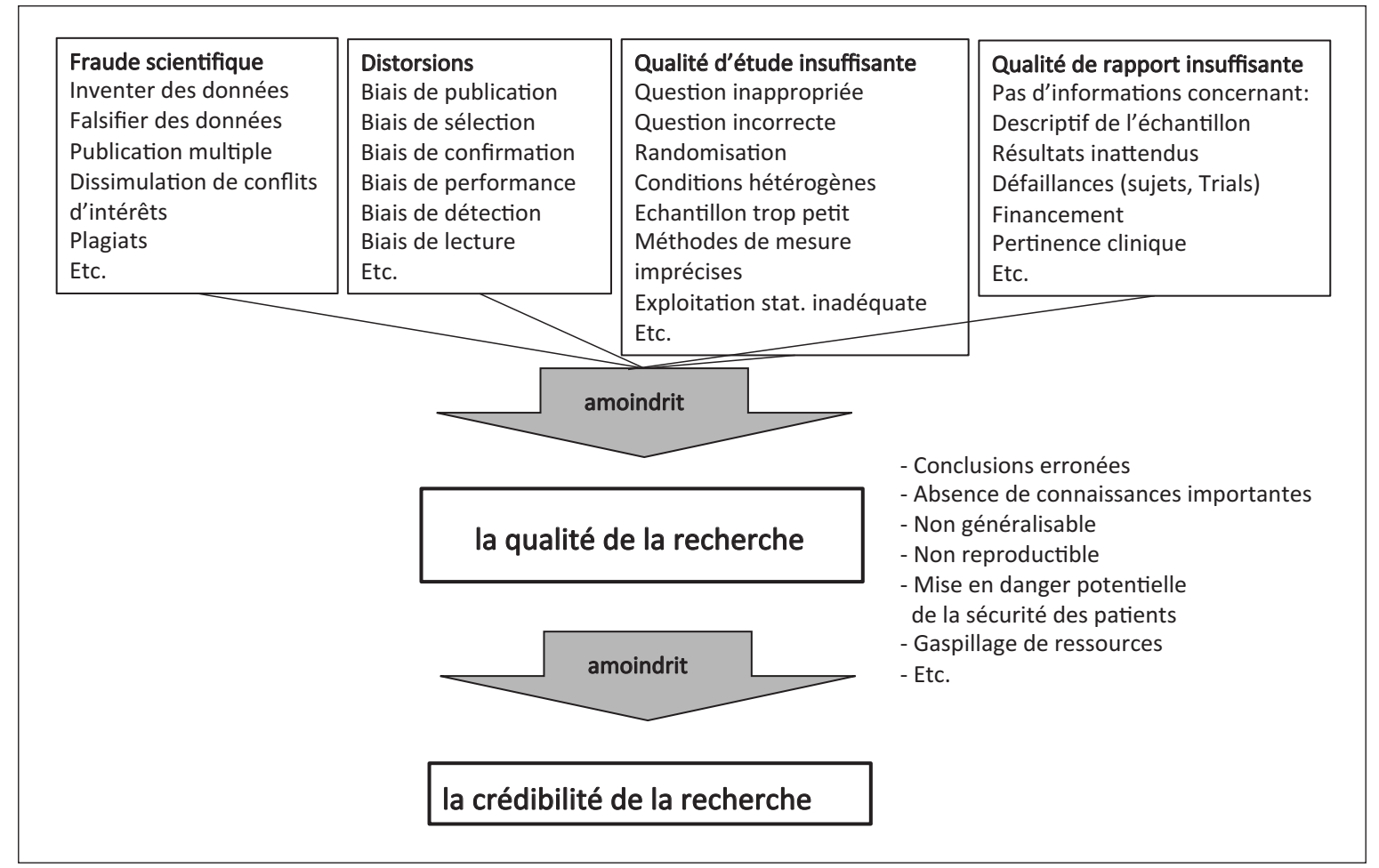

Figure 1. Représentation schématique de facteurs d'influence possibles sur la qualité de la recherche. 
Biais de publication

$50 \%$ des études ne sont pas publiées, notamment lorsque les résultats sont négatifs ou nonsignificatifs $[10,11]$. Souvent, les résultats publiés ne correspondent plus à la question initialement posée, ou bien certains résultats sont repris, modifiés ou abandonnés [20, 21]. Ce phénomène s'explique d'une part par le fait que des résultats significatifs et positifs (études de reproductibilité de résultats existants) font l'objet d'une meilleure évaluation et que leur publication paraît plus simple [20, 22]. D'autre part, l'industrie exerce une grande influence en sponsorisant les études mais en renonçant à la publication de certaines d'entre elles, comme l'indique l'étude de Jones et al. 2013 [23]. Selon ces derniers, les études financées par l'industrie sont moins fréquemment publiées que celles bénéficiant d'un autre financement. Par ailleurs, Jones et al. 2013 [23] ont montré que $29 \%$ des études de grande ampleur (>500 participants) n'ont pas été publiées. Il s'agirait selon eux d'une décision délibérée des sponsors car des études d'une telle ampleur n'auraient aucun problème à être publiées dans des revues de renom.

\section{Biais de confirmation}

Les résultats sont interprétés différemment et de manière subjective en fonction des intérêts, des connaissances et des préjugés du lecteur. Dans le cas le plus extrême, ce dernier privilégiera les informations qui confirment ses idées préconçues et, lors de l'utilisation ultérieure des données préalablement publiées, modifiera les résultats afin qu'ils "cadrent» avec son hypothèse. C'est ainsi que des erreurs se perpétuent à partir d'informations faussement interprétées.

\section{Biais de sélection}

Le biais de sélection traduit le fait que l'échantillon inclus dans l'étude n'a pas été sélectionné au hasard. Ce phénomène conduit à des "distorsions» ou des erreurs systématiques dans les résultats d'études.

\section{Biais de performance}

L'inégalité de traitement - délibérée ou non - des groupes de référence crée des conditions-cadres différentes.

\section{Biais de détection}

L'appartenance du chercheur à un groupe particulier influence son évaluation des résultats. Une enquête sur l'intérêt du jogging et du vélo auprès des groupes de référence respectifs (jogging/vélo), par exemple, ne sera pas impartiale si elle est menée par un biker passionné. Le risque est d'autant plus grand lorsque l'interprétation des résultats laisse une certaine marge d'appréciation et/ou dépend des interactions entre le sujet et le chercheur.

\section{Biais d'attrition}

Un biais d'attrition se traduit par le non-respect du protocole et la sortie prématurée de sujets d'étude: il peut arriver qu'un groupe respecte moins qu'un autre le protocole ou que ses sujets sortent de l'étude (en raison d'effets collatéraux par ex.).

D’autres distorsions sont définies dans un aperçu des différentes formes de biais comme le biais de la base de données, le biais de dissémination, le biais de décalage, le biais d'amplification des citations et le biais linguistique [24].

\section{Qualité insuffisante des études scientifiques}

Les mauvaises planification, réalisation et évaluation d'une étude scientifique ont un impact négatif sur sa qualité. C'est le cas par exemple lorsqu'une étude est menée sans que la littérature scientifique correspondante n'ait été préalablement passée en revue ou lorsque le type d'étude ne correspond pas à la question posée. Le nombre trop restreint de cas est également un problème fréquent qui diminue la pertinence statistique. Cela entraîne un manque de précision et de fiabilité des résultats. Par ailleurs, la méthode d'évaluation statistique choisie est souvent inappropriée. Ioannidis et al., 2005, déterminent, à l'aide d'une formule mathématique, les facteurs entravant la qualité des études scientifiques: petites études et grands effets, flexibilité dans le design, définitions, résultats et modèles analytiques [25]. Freedman et al., 2015 [9], montrent que 50\% des études ne sont pas reproductibles en raison d'échantillons trop petits et de lacunes méthodologiques.

\section{Qualité insuffisante des rapports d'études}

Les études font fréquemment l'objet de publications imprécises et incomplètes [16]. En outre, les études publiées sont souvent les seules informations disponibles pour évaluer la qualité des recherches accomplies. Si une publication est lacunaire, aucune évaluation fiable des résultats de l'étude ne peut être réalisée [16].

\section{Causes possibles de la qualité insuffisante de la recherche}

La mauvaise qualité d'une recherche scientifique dépend du comportement des chercheurs. Ces derniers peuvent, en effet, subir l'influence des facteurs suivants:

\section{Système de recherche et concurrence sur les publications}

Pour mesurer la contribution à l'excellence scienti- 


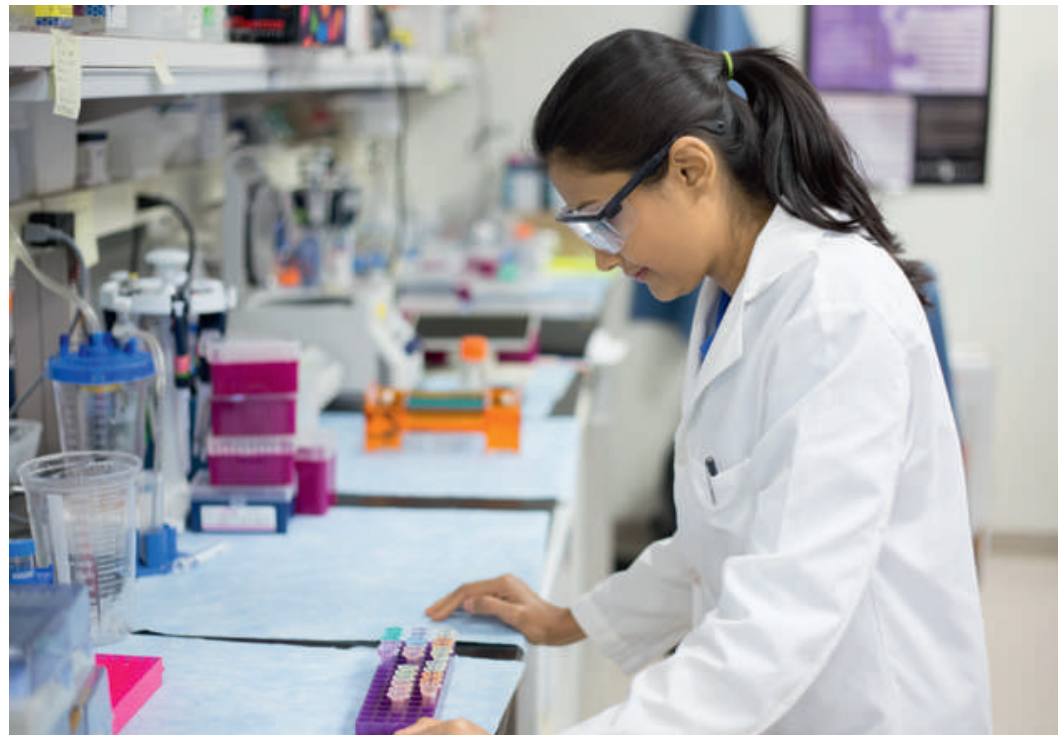

L'évidence scientifique requiert des résultats d'études fiables. Or la qualité de la recherche biomédicale fait toujours plus l'objet de critiques.

fique [26], il semblerait que le système de recherche actuel accorde davantage de poids au nombre de publications qu'au gain de connaissances. La compétition pour des postes universitaires, des fonds destinés à la recherche et la renommée obligent les chercheurs à publier le plus possible et le plus rapidement possible. Il est probable que ces incitatifs inhérents au système actuel aient un effet négatif sur la qualité de la recherche et conduisent au volume excessif actuel de publications.

\section{Dépendances financières et influence de l'industrie}

Les liens d'intérêt financiers influencent le comportement personnel ainsi que les estimations des chercheurs. Les entreprises pharmaceutiques renoncent, en outre, délibérément à la publication de certains résultats ou les publient sans respecter leur véracité [27]. En effet, dans de nombreux cas, les annonces publicitaires des revues spécialisées internationales ne correspondent pas aux résultats de la publication scientifique initiale sur ce sujet (par ex. exagération de l'effet thérapeutique, extension du domaine d'indication, reprise erronée des contenus de lignes directrices, minimisation d'effets secondaires, etc.) [27].

\section{Identifier les problèmes de qualité}

Une étude scientifique ne peut exclure une part de hasard et de variabilité. L'évaluation de la qualité de la recherche consiste à examiner toutes les étapes de la recherche - de l'évidence existante à la publication et à rechercher les points faibles, les erreurs, les omissions et les inadéquations.

\section{Contrôle critique et compétence méthodologique}

Les erreurs et la violation des principes fondamentaux de la bonne pratique scientifique sont généralement difficiles à détecter [28, 29].

L'évaluation positive d'une publication par les pairs n'est pas un gage automatique de la qualité de l'étude. En effet, les possibilités d'évaluation des études scientifiques se limitent aux informations comprises dans les publications. Une évaluation globale de la qualité des études exige à la fois une compétence méthodologique ainsi que l'accès aux données, aux protocoles d'études et autres documents importants.

\section{Instruments d'évaluation de la qualité de la recherche}

Divers instruments et listes de contrôle sont à disposition pour évaluer la qualité de certains aspects de la recherche. Pour gérer les principes de l'intégrité scientifique, plusieurs documents publics sont à disposition, par ex. la brochure "Intégrité dans le domaine scientifique» (Académies Suisses des Sciences) [17], les «Bases juridiques pour le quotidien du médecin» (FMH/ASSM) [30] et la directive «Collaboration corps médical - industrie» (ASSM) [31].

Pour évaluer le risque de biais, plusieurs instruments et listes de contrôle sont à disposition comme le "Cochrane collaboration tool for assessing risk of bias in RCTs 2011" [32]. Ces outils sont cependant limités dans la mesure où ils ne permettent d'évaluer que le contenu du rapport de recherche [16].

L'examen de la qualité d'un rapport de recherche doit permettre de déterminer si une publication contient toutes les informations pouvant rendre l'étude reproductible [33]. Le réseau EQUATOR (Enhancing the QUAlity and Transparency of health Research; www.equatornetwork.org) a récapitulé les principales lignes directrices pour les rapports de recherche (Reporting Guidelines).

\section{Aspect juridique}

L'ouverture d'une procédure en cas de fraude scientifique présumée incombe en premier lieu aux institutions de recherche dans lesquelles la fraude aurait été constatée. [17]. Si la fraude ne peut pas être exclue, l'organisation compétente en matière d'intégrité au sein de l'institution respective (médiateur, chargé de la protection de l'intégrité scientifique, instance d'enquête et 
de décision liée au cas) examine s'il y a violation de l'intégrité scientifique et quelles sont les sanctions qui doivent être prises (par ex. blâme ou avertissement écrit, réduction des fonds, blocage ou demande de restitution des fonds, exclusion, etc.). Le non-respect de dispositions légales (droit des brevets, législation sur les médicaments, sur la transplantation, etc.) doit déboucher sur une plainte ou une action en justice auprès des tribunaux nationaux compétents [30] et des sanctions financières et/ou pénales peuvent être prononcées.

\section{Mesures d'appui possibles à la qualité de la recherche}

\section{Enregistrement obligatoire des études}

L'obligation d'enregistrer les études permet de vérifier si la question initialement posée par une étude a été publiée ou de connaitre les raisons pour lesquelles l'étude a été stoppée. En Suisse, tout essai clinique doit être enregistré dans le Registre SNCTP (Swiss National Clinical Trials Portal).

\section{Accès aux protocoles d'étude, aux données et aux publications}

Pour encourager la transparence et la qualité, il serait primordial que les protocoles d'étude, les enregistrements de laboratoire et les données brutes soient accessibles au public. Par ailleurs, les publications publiques et les portails Open Access (par ex. PLOS The Public Library Of Science) devraient être encouragés. Les hautes écoles et les consortiums sont appelés à élaborer des solutions en vue du financement de publications en libre accès [34].

\begin{tabular}{|c|c|}
\hline Faux & Données falsifiées, fabriquées \\
\hline Données incohérentes & $\begin{array}{l}\text { Confirmation des soupçons de tiers envers } \\
\text { les données }\end{array}$ \\
\hline Erreurs & $\begin{array}{l}\text { Erreurs dans les données, détectées par } \\
\text { les auteurs }\end{array}$ \\
\hline Plagiat & $\begin{array}{l}\text { Publication de données ou de textes ayant déjà } \\
\text { été publiés par d'autres }\end{array}$ \\
\hline Recoupement & Publications multiples ou auto-plagiat \\
\hline $\begin{array}{l}\text { Objections juridiques ou réserves } \\
\text { en matière de propriété }\end{array}$ & $\begin{array}{l}\text { Publication d'éléments sans l'autorisation } \\
\text { des auteurs }\end{array}$ \\
\hline Ethique & Réserves sur la validité éthique de la recherche \\
\hline Notion d'auteur & Auteur contesté \\
\hline Rédacteur & Erreur de production ou d'administration \\
\hline
\end{tabular}

Source: Decullier et al. BMC Research Notes 2013, 6:238 [35].

\section{Assurance qualité}

Les institutions de recherche devraient disposer d'une instance chargée de contrôler le respect des règlements (Good Clinical Practice, Déclaration d'Helsinki, loi relative à la recherche sur l'être humain, etc.) et de mettre en place des mesures d'assurance qualité (audits, monitorage, etc.). Les experts et les rédacteurs de revues scientifiques doivent assumer leur responsabilité scientifique, contrôler avec soin et de manière critique les enregistrements de publications et les documents disponibles, et exiger d'éventuels dossiers supplémentaires.

Les maisons d'édition de revues scientifiques devraient définir des critères clairs et uniformes concernant la rétractation d'une étude. La diffusion et l'utilisation de portails Post Publication Review (par ex. PubPeer, Pub Med Commons) doivent être encouragées.

Une lecture critique de la littérature scientifique et l'évaluation de l'évidence de la recherche sont des aptitudes qui s'acquièrent. D'où l'importance de modèles, d'hôpitaux de formation et de cours pour étudiants et chercheurs. A titre d'exemple, un Massive Open Online Course (MOOC) intitulé Unethical decision making in organizations (www.coursera.com) est d'ores et déjà proposé.

Pour améliorer la qualité de la recherche et la pertinence des études, il serait essentiel de renforcer la coopération avec d'autres chercheurs du même domaine scientifique afin de mener des études de plus grande ampleur.

Il est en outre primordial d'encourager une recherche indépendante car les intérêts et les objectifs de l'industrie et des chercheurs ne convergent pas automatiquement. Par ailleurs, l'intérêt du patient doit être davantage pris en compte dans la recherche. Le nouveau programme spécial pour les essais cliniques lancés à l'initiative des chercheurs (Investigator Initiated Clinical Trials, IICT) du Fonds National Suisse FNS encourage la recherche clinique indépendante. Ce programme permet aux chercheurs de répondre à des questions présentant un intérêt pour les patients (www.snf.ch/fr/ pointrecherche/newsroom/Pages/news-150626-com munique-de-presse-nouveau-programme-rechercheclinique-independante.aspx).

\section{Evaluation d'études scientifiques}

Le système de recherche devrait accorder une place beaucoup plus importante à la qualité qu'elle ne le fait aujourd'hui. En effet, le nombre de publications ne reflète pas l'importance des recherches menées par un scientifique [26]. En revanche, les études et les publications devraient être évaluées selon des critères de qualité (reproductibilité, originalité, gain de connais- 
sances, etc.) en tenant compte des conflits d'intérêts financiers (avec déclassement éventuel de l'évidence). De manière générale, une plus grande valeur doit être accordée aux études menant à des résultats non positifs et non significatifs, et leur publication doit être facilitée.

\section{Rétractation d'une publication}

La rétractation d'une publication est l'une des sanctions possibles contre une étude de qualité médiocre. La plupart des publications sont retirées pour cause d'usage de faux, plagiat, publication multiple des mêmes données, etc. (cf. tableau 1) [35]. Le site www.retraction watch.com tient ainsi une liste des études retirées l'objectif étant de publier l'instance ayant rétracté la publication. Ainsi, les articles retirés ne seraient plus cités tout en restant accessibles. Les règles d'une rétractation ne sont pas définies uniformément [35].

\section{Conclusions}

La recherche implique un sens aiguisé des responsabilités. Une recherche de haute qualité exige une intégrité scientifique, c'est-à-dire honnêteté, exactitude, transparence, etc. Un chercheur doit faire preuve d'une grande motivation, de discipline, d'opiniâtreté et d'engagement, sans aucune garantie de réussite. Dès lors, rien d'étonnant de rencontrer des cas de fraude et d'usage de faux. Mais il est probable que d'autres facteurs jouent aussi un rôle plus important en matière de qualité: par ex. le fait que de nombreuses études ne sont pas publiées, que peu d'efforts sont déployés pour reproduire des études, que de nombreuses questions de la recherche clinique ne sont pas pertinentes pour les patients. Le système de valeurs de la recherche académique doit être modifié car il faut beaucoup de temps avant d'apporter une contribution importante à la recherche scientifique. Il serait donc judicieux d'évaluer les études scientifiques et la recherche à la lumière de leur qualité, et non sur la base du nombre de publications.

Non seulement une recherche erronée représente un formidable gaspillage de ressources humaines et financières, mais conduit également à de fausses conclusions, et donc à une mise en danger de la sécurité des patients. La médecine basée sur les preuves notamment (efficacité démontrée de manière empirique) est déterminante pour la pratique quotidienne, que ce soit par l'utilisation de directives ou dans la formation prégraduée, postgraduée et continue. Les médecins sont responsables du traitement de leurs patients. Ils doivent par conséquent porter un regard critique sur la recherche médicale et tenir compte des résultats obtenus, notamment dans leur mise en application de la médecine basée sur les preuves.

La crédibilité de la science exige notamment plus de recherche indépendante, la prise en compte de l'intérêt du patient, une communication transparente, indépendamment des résultats, et un changement du système de valeurs académique. Les médecins chercheurs en particulier peuvent contribuer à cette mutation en s'engageant pour une recherche de haute qualité.

\section{Crédit photo}

(c) Nandyphotos | Dreamstime.com 


\section{Références}

1 SEFRI. Analyse bibliométrique de la recherche scientifique en Suisse 1981-2011. www.sbfi.admin.ch/dokumentation/00335/01740/index.html?lang=fr, 2014

2 SBF. Switzerland - The place for education, research and innovation. www.sbfi.admin.ch/dokumentation/01816/index. html?lang=en, 2015.

3 Die Forschung in der Glaubwürdigkeitskrise Tages-Anzeiger, 2015; www.tagesanzeiger.ch/wissen/medizin-und-psychologie/ Die-Forschung-in-der-Glaubwuerdigkeitskrise-/story/24741498.

4 Loder E, Godlee F, Barbour V, Winker M. Restoring the integrity of the clinical trial evidence base. BMJ. 2015; 346:f3601.

5 Roston M. Retracted Scientific Studies: A Growing List. New York Times; 2015. www.nytimes.com/interactive/2015/05/28/science/ retractions-scientific-studies.html? $\mathrm{r}=0$.

6 Ioannidis JP. How to make more published research true. PLoS Med, 2014; 11: e1001747.

7 Every-Palmer S, Howick J. How evidence-based medicine is failing due to biased trials and selective publication. J Eval Clin Pract. 2014; 20:908-14.

8 Obrist R. Only conflicts of interest? Swiss Med Wkly, 2015; 145:w14120.

9 Freedman LP, Cockburn IM, Simcoe TS. The Economics of Reproducibility in Preclinical Research. PLoS Biol, 2015; 13: e1002165.

10 Easterbrook PJ, Berlin JA, Gopalan R, Matthews DR. Publication bias in clinical research. Lancet. 1991;337:867-72.

11 Rising K, Bacchetti P, Bero L. Reporting bias in drug trials submitted to the Food and Drug Administration: review of publication and presentation. PLoS Med. 2008;5:e217; discussion e217.

12 Korevaar DA, Wang J, van Enst WA, Leeflang MM, Hooft L, Smidt N, Bossuyt PM. Reporting diagnostic accuracy studies: some improvements after 10 years of STARD. Radiology. 2015; 274:781-9.

13 Muir Gray JA. Medical Journal can be less biased. In book: Better doctors better patients decisions: Envisioning health care 2020. Cambridge. MA: MIT Press; 2011

14 Moher D, Hopewell S, Schulz KF, Montori V, Gotzsche PC, Devereaux PJ, Elbourne D, Egger M, Altman DG. CONSORT 2010 Explanation and Elaboration: Updated guidelines for reporting parallel group randomised trials. J Clin Epidemiol. 2010;63:e1-37.

15 Vandenbroucke JP, von Elm E, Altman DG, Gotzsche PC, Mulrow $\mathrm{CD}$, Pocock SJ, Poole C, Schlesselman JJ, Egger M. Strengthening the Reporting of Observational Studies in Epidemiology (STROBE) explanation and elaboration. Epidemiology. 2007;18:805-35.

16 Blümle A, Von Elm E, Antes G, Meerpohl JJ. Messung und Bewertung der Studienqualität und Berichtsqualität. Z. Evid. Fortbild. Qual. Gesundh. wesen. (ZEFQ). 2014;108:495-503.

17 Académie suisse des sciences. Intégrité dans le domaine scientifique. 2008.
18 Palazzo G, Krings F, Hoffrage U. Ethical Blindness. J Bus Ethics. 2012;109:323-38.

19 Juni P, Altman DG, Egger M. Systematic reviews in health care: Assessing the quality of controlled clinical trials. BMJ. 2001;323:42-6.

20 Dwan K, Gamble C, Williamson PR, Kirkham JJ. Systematic review of the empirical evidence of study publication bias and outcome reporting bias - an updated review. PLoS One. 2013;8:e66844.

21 Kirkham JJ, Dwan KM, Altman DG, Gamble C, Dodd S, Smyth R, Williamson PR. The impact of outcome reporting bias in randomised controlled trials on a cohort of systematic reviews. BMJ. 2010;340:c365.

22 Melander H, Ahlqvist-Rastad J, Meijer G, Beermann B. Evidence b(i) ased medicine - selective reporting from studies sponsored by pharmaceutical industry: review of studies in new drug applications. BMJ. 2003; 326:1171-3.

23 Jones CW, Handler L, Crowell KE, Keil LG, Weaver MA, Platts-Mills TF. Non-publication of large randomized clinical trials: cross sectional analysis. BMJ. 2013;347:f6104

24 Song F, Parekh S, Hooper L, Loke YK, Ryder J, Sutton AJ, Hing C, Kwok CS, Pang C, Harvey I. Dissemination and publication of research findings: an updated review of related biases. Health Technol Assess. 2010;14: iii, ix-xi, 1-193.

25 Ioannidis JP. Why most published research findings are false. PLoS Med. 2005;2:e124.

26 Binswanger M. Der Publikationswettbewerb in der Forschung: Arroganzen, Ignoranzen, Redundanzen. LIFIS Online. 2011; www. leibniz-institut.de

27 Kaiser T, Ewers H, Waltering A, Beckwermert D, Jennen C, Sawicki PT. Sind die Aussagen medizinischer Werbeprospekte korrekt? arznei-telegramm. 2004;35.

$28 \mathrm{Krumholz} \mathrm{HM}$. Open science and data sharing in clinical research basing informed decisions on the totality of the evidence. Circ Cardiovasc Qual Outcomes. 2012;5:141-2.

29 Fröhlich G. Peer Review und Abweisungsraten. Lehre \& Forschung. 2007;6:338-39.

30 FMH/ASSM. Bases juridiques pour le quotidien du médecin. 2013; chapitre 6 Recherche sur l'être humain.

31 ASSM. Collaboration corps médical - Industrie. 2013.

32 Higgins JP, Altman DG, Gotzsche PC, Juni P, Moher D, Oxman AD, Savovic J, Schulz KF, Weeks L, Sterne JA. The Cochrane Collaboration's tool for assessing risk of bias in randomised trials. BMJ. 2011;343:d5928.

33 Golub RM, Fontanarosa PB. Researchers, readers, and reporting guidelines: writing between the lines. Jama. 2015;313:1625-6.

34 Aguzzi A. Scientific publishing in the times of open access. Swiss Med Wkly. 2015;145:w14118

35 Decullier E, Huot L, Samson G, Maisonneuve H. Visibility of retractions: a cross-sectional one-year study. BMC Res Notes. 2013;6:238. 